\title{
Recent trends of HER-2 examination and a new strategy of trastuzumab therapy for primary and metastatic breast cancer
}

\author{
Masafumi Kurosumi
}

Published online: 10 September 2009

(C) The Japanese Breast Cancer Society 2009

Recently, therapeutic strategies for patients with breast cancer have made rapid advances since the introduction of molecular-targeting therapy using trastuzumab. At the same time, examination of HER-2 status using the IHC and ISH methods has become an established and accepted procedure for selection of breast cancer patients who will likely respond to trastuzumab. Recently, large-scale clinical studies have confirmed the effectiveness of anti-cancer therapies incorporating trastuzumab for metastatic as well as primary breast cancer.

In the first review, Dr. Kurosumi describes recent trends of HER-2 testing for breast cancer. The process of HER-2 testing and the algorithm employed are explained; tumors showing a category score of " $3+$ " by immunohistochemistry (IHC) and/or a signal ratio of HER-2/CEP17 higher than 2.0 by fluorescence in situ hybridization (FISH) are thought to be potentially responsive to trastuzumab therapy. In addition, a new guideline of HER-2 testing proposed by the ASCO/CAP group in 2007 is presented, and new ISH methods such as BDISH and dc-CISH are introduced. On the other hand, it is emphasized that quality control of HER-2 testing is thought to be necessary.

In the second review, Dr. Iwata discusses the effectiveness of adjuvant trastuzumab therapy. Clinical trials of adjuvant trastuzumab have indicated that it improves disease-free as well as overall survival after, or in combination with, adjuvant chemotherapy. In addition, the importance of trastuzumab as a potential standard therapy for HER2-positive breast cancer patients worldwide is described. Consideration is also given to many uncertain issues related to trastuzumab adjuvant therapy, such as the duration of therapy, eligibility of elderly women, and its applicability for smaller tumors measuring $1 \mathrm{~cm}$ or less.

In the third review, Dr. Tokuda discusses the role of trastuzumab in the management of HER2-positive metastatic breast cancer. Trastuzumab is described as one of the key drugs in the strategy of treatment for HER2-positive breast cancer, and some HER2-positive patients have achieved long survival with a complete response to trastuzumab therapy. In HER2-positive metastatic breast cancer, a combination of trastuzumab with cytotoxic drugs is markedly effective, and major guidelines now recommend trastuzumab as a key drug for the management of HER2positive metastatic breast cancer. Attempts to find some answers to problems associated with trastuzumab therapy are also discussed on the basis of recent evidence.

This special project is particularly important for understanding recent information related to HER-2 testing and the effectiveness of trastuzumab for both metastatic breast cancer and in an adjuvant setting. 